\title{
Evaluation of vitamin D3 in patients of polycystic ovary syndrome and their correlation
}

\section{Rooplekha Chauhan, Sonal Sahani*, Ankita Garg}

Department of Obstetrics and Gynecology, Netaji Subhash Chandra Bose, Medical College Jabalpur, Madhya Pradesh, India

Received: 06 March 2017

Accepted: 01 April 2017

*Correspondence:

Dr. Sonal Sahani,

E-mail: ankitagarg2411@gmail.com

Copyright: ( ) the author(s), publisher and licensee Medip Academy. This is an open-access article distributed under the terms of the Creative Commons Attribution Non-Commercial License, which permits unrestricted non-commercial use, distribution, and reproduction in any medium, provided the original work is properly cited.

\section{ABSTRACT}

Background: Polycystic ovary syndrome (PCOS) is enigma faced by young females since the onset of urbanization. As on day, PCOS is the most common endocrine disorder in the women of reproductive age affecting 4-10\%. This study done to measure serum vitamin D3 levels of PCOS patients and to identify its association with factors associated with various symptoms and signs in PCOS.

Methods: Institutional based prospective observational study conducted on 70 patients of PCOS, both suspected and already diagnosed, attending gynecology OPD of Department of obstetrics and gynecology of NSCB Medical College Jabalpur from 1 March 2015 to 31 August 2016. Diagnosis of PCOS was made by Rotterdam 2003 criteria. A detailed assessment was done and preformed proforma was filled. Serum Vitamin D3 levels were estimated by standard lab technique 'Liquid Chromatography Tandem Mass Spectrometry' and categorized into vitamin D3 sufficient >30ng/dl, insufficient 20-29ng/dl and deficient $<20 \mathrm{ng} / \mathrm{dl}$. Categorical data was coded numerically before the analysis. For comparison of frequency distribution chi square test or fisher's exact test were applied as appropriate.

Results: Prevalence of Vitamin D3 deficiency in patients of PCOS was found to be $84.3 \%$.

Conclusions: The present study concludes that there is correlation between vitamin D3 and PCOS, screening and correction of vitamin D3 deficiency may prevent PCOS and its manifestations. Hence, it is speculated that improvement of vitamin D3 levels at a younger age can contribute to prevention of PCOS.

Keywords: Polycystic ovary syndrome, Rotterdam criteria, Vitamin D3 deficiency

\section{INTRODUCTION}

Polycystic ovary syndrome (PCOS) is the most common endocrine disorder of women of reproductive age affecting about $4-10 \%$ of all women. ${ }^{1,2}$ Classical features of PCOS include infertility, hyperandrogenism, truncal obesity, abnormal glucose metabolism, insulin resistance (IR), hirsutism, and acne vulgaris, but diagnostic criteria include;

- Chronic oligomenorrhea or amenorrhea

- Evidence of androgen excess clinical or biochemical or

\section{- Polycystic ovaries}

Stress is one of the important factors in the aetiology of PCOS; it is seen in patients of PCOS from the young age till old. There are three major sets of diagnostic criteria for the diagnosis of PCOS

- National Institutes of Health (NIH) Bethesda, Maryland, in 1990: Chronic anovulation and clinical and biochemical signs of hyperandrogenism. (both criteria must be present, and other diagnoses must be excluded). ${ }^{3}$ 
- Rotterdam criteria in 2003: Oligo-ovulation, clinical and biochemical signs of hyperandrogenism and Polycystic appearing ovaries on imaging (any two of the above three must be present and exclusion of other androgen excess or related disorders). ${ }^{4}$

- Androgen excess and PCOS (AE-PCOS) society in 2009: Hyperandrogenism includes hirsutism and ovarian dysfunction including oligo-anovulation and polycystic appearing (exclusion of other androgen excess or related disorders). ${ }^{5}$

\section{Vitamin D}

Vitamin D is a steroid hormone that is primarily known for its role in skeletal health and calcium homeostasis, but its deficiency has been related to a number of nonskeletal disorders including obesity, insulin dysregulation, dyslipidaemia, hypertension, cardiovascular diseases, autoimmune diseases, and even cancers. ${ }^{6}$

\section{Vitamin D deficiency in PCOS}

Vitamin D deficiency is common in women with PCOS, with the $67-85 \%$ of women with PCOS having serum concentrations of 25-hydroxy vitamin D (25OHD) $<20$ $\mathrm{ng} / \mathrm{ml}^{7}$ It is newly accepted concept that the metabolic changes in PCOS are related to dysfunction of vitamin D and calcium metabolism, which is important in follicular development and normal glucose metabolism. ${ }^{8,9}$ Multiple studies have shown that PCOS patients, particularly if obese, have lower serum vitamin D levels. ${ }^{10-13}$ and have been associated with hyperandrogenism, metabolic syndrome, insulin resistance, and increased body mass index (BMI). ${ }^{10,12-15}$

Many studies have been conducted to validate these associations with vitamin D but limited evidence has shown that vitamin D and calcium metabolism affects oocyte maturation and production of androgens.

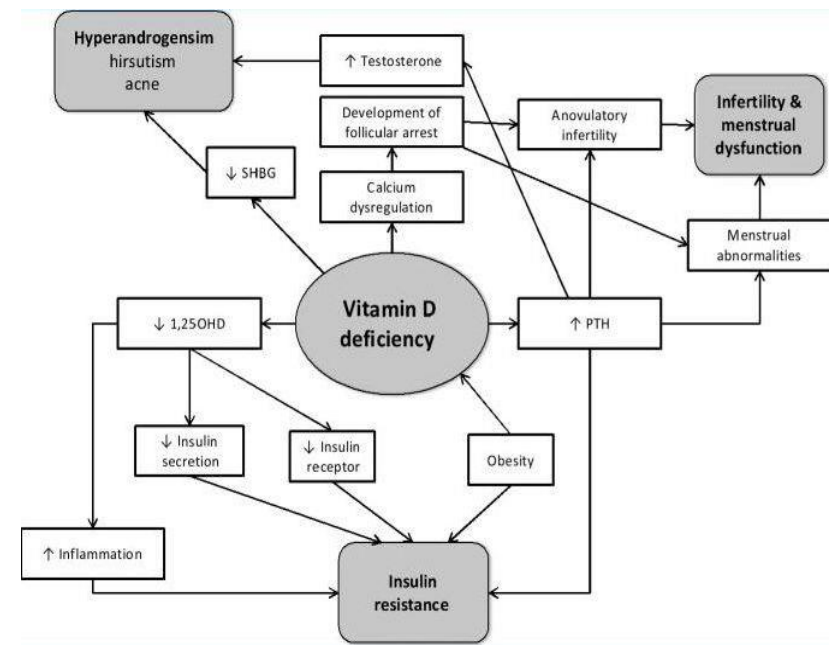

Figure 1: The Role of vitamin D deficiency in the pathology of PCOS.
One research revealed that 1,25-dihydroxyvitamin D mediates an enzyme known as aromatase which converts androgen derivatives to estrogen in granulosa cells of the ovaries. $^{16}$ Therefore, dysfunction in this mechanism affects the menstrual cycle and ovulation by altering the hormone levels mediated. To validate some of these outcomes, researchers have also experimented in treating PCOS patients with vitamin D and calcium supplementation. This observational study also studied the correlation between vitamin D levels and PCOS and its various parameters, which are depicted in Figure $1 .{ }^{17}$

\section{METHODS}

The present study was hospital based prospective observational study and was conducted on patients of PCOS, both suspected and already diagnosed (by Rotterdam criteria), attending gynecology OPD of Department of obstetrics and gynecology of NSCB Medical College Jabalpur from 1 March 2015 to 31 August 2016.

Approval from the institutional ethical committee was taken before conducting the study. Total 156 patients of PCOS were counselled and out of these 70 patients consented to participation in study and investigations.

\section{Inclusion criteria}

All newly suspected and diagnosed cases of PCOS.

\section{Exclusion criteria}

- Any diagnosed case of PCOS who was on and had history of taking vitamin D and calcium supplement within period of one year.

- Diagnosed cases of PCOS who was under treatment and recovered with treatment (medical and surgical).

- Patients who were not willing to take part in the study.

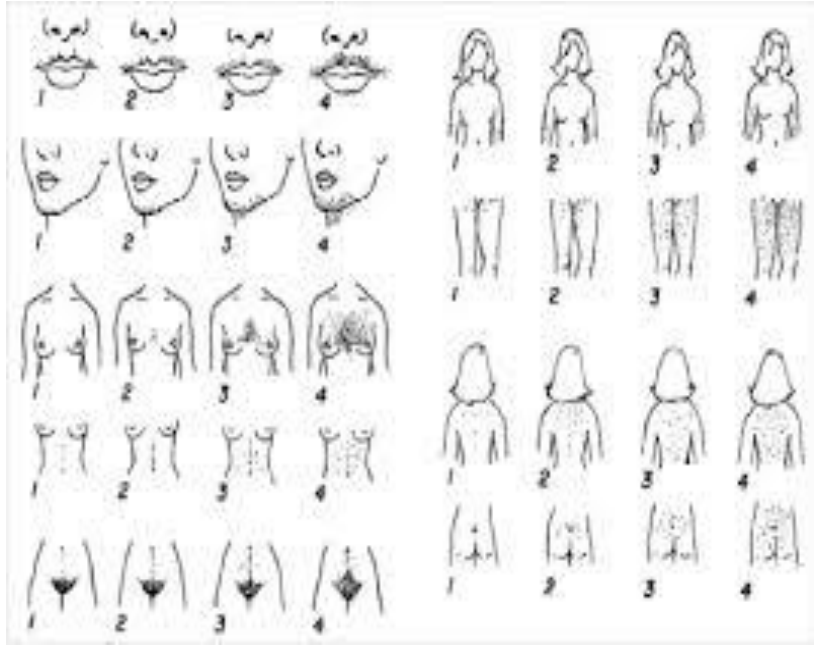

Figure 2: Ferrimann-Gallwey hirsutism scoring system. 
All patients were informed about the nature of disease, and aims of study and verbal consent was taken. Diagnosis of PCOS was made by Rotterdam 2003 criteria. A detailed assessment was done and performed proforma was filled. Demographic data was collected, relevant history and chief complaints were noted. Any comorbidities like diabetes, hypertension and thyroid dysfunction present or absent in subjects was mentioned. Lifestyle of subjects was assessed by physical activity which was graded as 1) active, 2) moderate and 3) sedentary activity. Standard anthropometric data height, weight, BMI, waist circumference, hip circumference was measured. Waist-hip ratio (WHR) was calculated and classified according to WHO guided health risks into low, moderate and high risks as <0.80, 0.80-0.85 and $>0.85$ respectively. BMI was classified by WHO classification and cases were divided into non-obese $(\mathrm{BMI}<25)$ and obese (BMI >25) then mean was calculated and compared. Acne was graded in 4 grades according to severity.

Hirsutism was quantified according to modified Ferriman-Gallwey-Score which was filled by subjects in the chart Figure 2 and total score was calculated and quantified as <8 non-hirsutisms, hirsutism >8-15 and overt hirsutism $>15$. Sites involved for acanthosis nigricans (AN) was noted neck, axilla, elbow, knuckles and knees. AN was graded into 4 grades: Grade 1-visible on inspection, Grade 2-limited to base of skull, Grade 3extending to lateral margin of neck, Grade 4-extending anteriorly. Serum Vitamin D3 was estimated by standard laboratory technique 'Liquid Chromatography Tandem Mass Spectrometry' and classified according to reference range.

Vitamin D3

Vitamin D3 sufficient

Vitamin D3 insufficient

Vitamin D3 deficient

\section{Levels}

(25[OH]D $\geq 30 \mathrm{ng} / \mathrm{ml})$

(25[OH]D $20-29 \mathrm{ng} / \mathrm{ml})$

$(25[\mathrm{OH}] \mathrm{D}<20 \mathrm{ng} / \mathrm{ml}]$

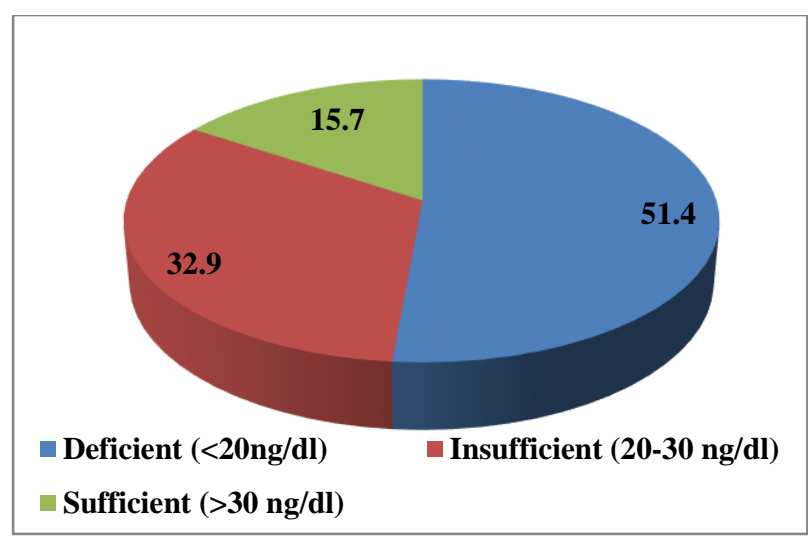

Figure 3: Distribution of vitamin D3 levels in cases of PCOS.

Majority of cases $38.4 \%$ were in younger age group 1620 years followed by $30 \% 21-25$ years and $27.1 \%$ 26-30 years, out of them $83.9 \%, 94.1 \%$ and $46.7 \%$, respectively were vitamin D3 deficient (Figure 4).

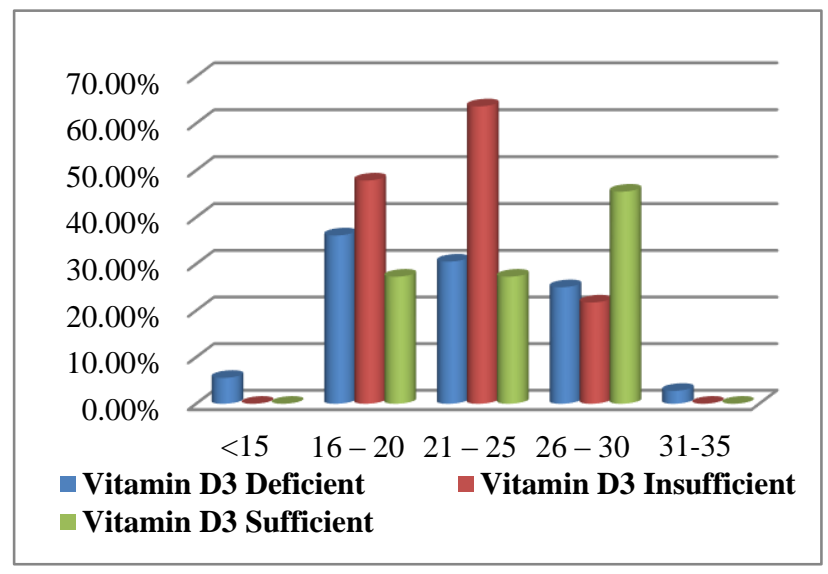

Figure 4: Age wise distribution of vitamin D3 level of cases of PCOS.

Total 70 PCOS patients mainly had complaints of oligomenorrhea $(82.9 \%)$, weight gain $(70 \%)$, acne (52.9\%), hirsutism (50\%), AN (32.9\%) and corresponding vitamin D3 deficiency were noted as $86.2 \%, 83.7 \%, 81 \%, 88.5 \%$ and $95.7 \%$ respectively (Table 1).

Out of 70 PCOS patients, $84.3 \%$ (59) had serum vitamin D3 levels less than required i.e. $30 \mathrm{ng} / \mathrm{dl}$ and only $15.7 \%$ (11) had sufficient levels (Figure 3).

Table 1: Distribution of vitamin D3 levels of cases of PCOS according to their presenting complaints.

\begin{tabular}{|lllll|}
\hline Presenting complaints & Vitamin D3 Deficient & Vitamin D3 Insufficient & Vitamin D3 Sufficient & Total \\
\hline Oligomenorrhea & $29(50 \%)$ & $21(36.2 \%)$ & $8(13.8 \%)$ & 58 \\
\hline Amenorrhoea & $12(57.1 \%)$ & $7(33.3 \%)$ & $2(9.5 \%)$ & 21 \\
\hline Menorrhagia & $11(52.4 \%)$ & $7(33.3 \%)$ & $3(14.3 \%)$ & 21 \\
\hline Hirsutism & $18(51.4 \%)$ & $13(37.1 \%)$ & $4(11.4 \%)$ & 35 \\
\hline Acne & $17(45.9 \%)$ & $13(35.1 \%)$ & $7(18.9 \%)$ & 37 \\
\hline Acanthosis nigricans & $14(60.9 \%)$ & $8(34.8 \%)$ & $1(4.3 \%)$ & 23 \\
\hline Alopecia & $10(66.7 \%)$ & $3(20 \%)$ & $2(13.3 \%)$ & 15 \\
\hline Infertility & $15(62.5 \%)$ & $6(25 \%)$ & $3(12.5 \%)$ & 24 \\
\hline Weight gain & $24(49 \%)$ & $17(34.7 \%)$ & $8(16.3 \%)$ & 49 \\
\hline
\end{tabular}

Chi square test was applied and $\mathrm{P}$ value was insignificant for all variables 
Out of 28 married patients $85.7 \%$ (24) had chief complaint of infertility and out of them $87.5 \%$ were vitamin D3 deficient (Table 1).

Statistically significant direct correlation was found between physical activity and vitamin D3 levels in PCOS patients, $40 \%$ physically active, $84.5 \%$ moderately active and $95 \%$ sedentary patients were vitamin D3 deficient (Table 2).

Inverse statistically significant correlation was found between hirsutism score (Ferriman-Gallwey-Score) and corresponding vitamin D3 level, $88.1 \%$ hirsute and 100\% overt hirsute patients were vitamin D3 deficient compared to $71.8 \%$ non-hirsute (Table 3 ).

41 out of 70 patients had acne and $34(82.9 \%)$ were vitamin D3 deficient, grade1 $85.7 \%$, grade $283.3 \%$, grade $380 \%$ and grade $460 \%$. Inverse correlation was found between vitamin D3 and severity of AN. 25 out of 70 had AN of axilla and $100 \%$ were vitamin D3 deficient which was found statistically significant (Table 4).

Table 2: Distribution of vitamin D3 levels of cases of PCOS according to physical activity.

\begin{tabular}{|lllll|} 
Physical Activity & Vitamin D3 Deficient & Vitamin D3 Insufficient & Vitamin D3 Sufficient & Total \\
Active & $1(20 \%)$ & $1(20 \%)$ & $3(60 \%)$ & $5(7.1 \%)$ \\
\hline Moderate & $22(48.9 \%)$ & $16(35.6 \%)$ & $7(15.5 \%)$ & $45(64.3 \%)$ \\
\hline Sedentary & $13(65 \%)$ & $6(30 \%)$ & $1(5 \%)$ & $20(28.6 \%)$ \\
Total & $\mathbf{3 6}$ & $\mathbf{2 3}$ & $\mathbf{1 1}$ & $\mathbf{7 0}$ \\
\hline
\end{tabular}

$\chi 2=9.836, \mathrm{P}$ value $<0.05$ (significant)

Table 3: Distribution of vitamin D3 levels of cases of PCOS according to the hirsutism score.

\begin{tabular}{|lllll|} 
Hirsutism score & Vitamin D3 Deficient & Vitamin D3 Insufficient & Vitamin D3 Sufficient & Total \\
\hline Non-hirsutism & $7(31.8 \%)$ & $9(40.9 \%)$ & $6(27.3 \%)$ & $22(31.4 \%)$ \\
\hline Hirsutism & $27(64.3 \%)$ & $10(23.8 \%)$ & $5(11.9 \%)$ & $42(60 \%)$ \\
\hline Overt hirsutism & $2(33.3 \%)$ & $4(66.7 \%)$ & $0(0 \%)$ & $6(8.6 \%)$ \\
\hline Total & $\mathbf{3 6}$ & $\mathbf{2 3}$ & $\mathbf{1 1}$ & $\mathbf{7 0}(\mathbf{1 0 0} \%)$ \\
\hline
\end{tabular}

$\chi 2=10.146, \mathrm{P}$ value $<0.05$ (significant)

Table 4: Distribution of vitamin D3 levels of cases of PCOS according to the presence of acanthosis Nigricans.

\begin{tabular}{|ll|lll|}
\hline Acanthosis nigricans & Vitamin D3 Deficient & Vitamin D3 Insufficient & Vitamin D3 Sufficient & Total \\
\hline Neck & $16(50 \%)$ & $13(40.6 \%)$ & $3(9.4 \%)$ & 32 \\
\hline Axilla & $15(60 \%)$ & $10(40 \%)$ & $0(0 \%)$ & 25 \\
\hline Elbow & $12(75 \%)$ & $4(25 \%)$ & $0(0 \%)$ & 16 \\
\hline Knuckles & $10(58.8 \%)$ & $7(41.2 \%)$ & $0(0 \%)$ & 17 \\
\hline Knees & $12(75 \%)$ & $4(25 \%)$ & $0(0 \%)$ & 16 \\
\hline
\end{tabular}

Chi square test was applied and $\mathrm{P}$ value was insignificant for all variables except acanthosis Nigricans axilla $\chi^{2}=7.271$, $\mathrm{P}$ value $<0.05$ (significant)

Table 5: Distribution of vitamin D3 levels of cases of PCOS according to the acanthosis Nigricans grading of neck.

\begin{tabular}{|lllll}
\hline $\begin{array}{l}\text { Acanthosis nigricans } \\
\text { grading neck }\end{array}$ & Vitamin D3 deficient & Vitamin D3 insufficient & Vitamin D3 sufficient & Total \\
\hline Grade 0 & $14(36.8 \%)$ & $13(34.2 \%)$ & $11(28.9 \%)$ & $38(54.3 \%)$ \\
\hline Grade 1 & $5(41.6 \%)$ & $5(41.6 \%)$ & $2(16.7 \%)$ & $12(17.1 \%)$ \\
\hline Grade 2 & $7(77.7 \%)$ & $2(22.2 \%)$ & $0(0 \%)$ & $9(12.8 \%)$ \\
\hline Grade 3 & $5(71.4 \%)$ & $2(28.6 \%)$ & $0(0 \%)$ & $7(10 \%)$ \\
Grade 4 & $4(100 \%)$ & $0(0 \%)$ & $0(0 \%)$ & $4(5.7 \%)$ \\
\hline Total & & & & $\mathbf{7 0}(\mathbf{1 0 0 \%})$ \\
\hline
\end{tabular}

$\chi 2=21.9783, \mathrm{P}$ value $<0.05$ (significant)

On comparing AN grading of neck with serum vitamin D3 levels, there was inverse statistically significant correlation between them, grade $183.2 \%$, grade $2,3,4$ were $100 \%$ deficient compared to $71 \%$ deficiency in grade 0 (who did not have AN) (Table 5). In this study mean value of vitamin D3 in obese (BMI $>25)$ and nonobese (BMI <25) were 16.52ng/dl and 22.50ng/dl respectively on applying t-test difference was statistically significant (Table 6, Figure 5). 
Heath risks according to waist-hip ratio had statistically significant inverse correlation with vitamin D3 levels, moderate risk (WHR 0.8-0.85) 75\% and high risk (WHR $>0.85) \quad 90.2 \%$ patients were vitamin D3 deficient compared to low risk $(<0.80) 33.3 \%$ (Table 7).

Table 6: Comparision of mean vitamin D3 levels of obese and non-obese cases of PCOS (classified according to $\mathrm{BMI})$.

\begin{tabular}{|lcll|}
\hline BMI & N & $\begin{array}{l}\text { Mean vitamin } \\
\text { D3 level }\end{array}$ & $\begin{array}{l}\text { Standard } \\
\text { Deviation }\end{array}$ \\
\hline $\begin{array}{l}\text { Non-obese } \\
\text { (BMI <25) }\end{array}$ & 33 & 22.50 & 9.33 \\
\hline $\begin{array}{l}\text { Obese } \\
(\text { BMI > 25) }\end{array}$ & 37 & 16.52 & 8.76 \\
\hline
\end{tabular}

t-test $=2.76 \mathrm{P}$ value $=0.0074(<0.05)($ significant $)$

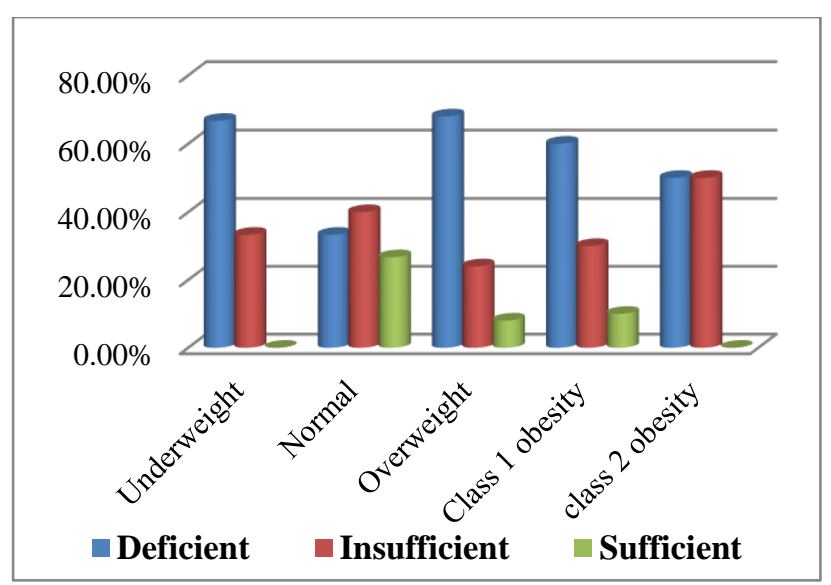

Figure 5: Distribution of vitamin D3 levels of cases of PCOS according to the basic metabolic rate (WHO).

Table 7: Distribution of vitamin D3 levels of cases of PCOS according to the waist-hip ratio (WHO).

\begin{tabular}{|lllll|}
\hline Waist - Hip Ratio & Vitamin D3 Deficient & Vitamin D3 Insufficient & Vitamin D3 Sufficient & Total \\
\hline Low risk $(<0.8)$ & $0(0 \%)$ & $1(33.3 \%)$ & $2(66.7 \%)$ & $3(4.3 \%)$ \\
\hline $\begin{array}{l}\text { Moderate risk } \\
(0.8-0.85)\end{array}$ & $6(37.5 \%)$ & $6(37.5 \%)$ & $4(25 \%)$ & $16(22.9 \%)$ \\
\hline High risk $(>0.85)$ & $30(58.8 \%)$ & $16(31.4 \%)$ & $5(9.8 \%)$ & $51(72.8 \%)$ \\
\hline Total & $\mathbf{3 6}$ & $\mathbf{2 3}$ & $\mathbf{1 1}$ & $\mathbf{7 0}(\mathbf{1 0 0 \%})$ \\
\hline
\end{tabular}

$\chi^{2}=9.796, \mathrm{P}$ value $<0.05$ (significant)

\section{DISCUSSION}

Out of 70 PCOS women who were enrolled in GOPD of NSCB Medical college $84.3 \%$ were found to have Vitamin D3 levels less than required. Figurová $\mathbf{J}$ et al, in their study 'Prevalence of vitamin D deficiency in Slovak women with PCOS and its relation to metabolic and reproductive abnormalities' found that the prevalence of vitamin $\mathrm{D}$ deficiency was high and similar in both groups, 79 of 99 PCOS women $(80 \%)$ and 46 of 66 control group subjects $(70 \%)(\mathrm{p}=0.138) .{ }^{18}$

Li HW et al, in an observational study 2011 including 25 women with PCOS and 27 controls to the prevalence of vitamin D deficiency in PCOS women in Scotland found the majority of PCOS subject's $n=18,72 \%$ were found to be vitamin $\mathrm{D}$ deficient. ${ }^{15}$ In an observational study, involving 206 PCOS women were studied by Wher et al, wherein $72.8 \%$ (150 women) of PCOS population demonstrated vitamin D deficiency. ${ }^{12}$

Majority of cases in this study had complaint of oligomenorrhea i.e. $82.9 \%$ (58) out of total 70 cases and $86.2 \%$ of them were noted with vitamin D3 deficiency. In one interventional randomized trail study by Rashidi B, et al, 60 infertile PCOS women were enrolled who had history of oligomenorrhea/amenorrhea during last 6 months prior to study. ${ }^{19}$ It was concluded that calcium- vitamin $\mathrm{D}$ along with metformin could be effective for the treatment of anovulation and oligomenorrhea in PCOS patients.

In our study $40 \%$ physically active, $84.5 \%$ moderately active and $95 \%$ sedentary PCOS patients were vitamin D3 deficient and this was found statistically significant. In study, done by Holick $F$ et al, concluded various skeletal and non-skeletal consequences of vitamin $\mathrm{D}$ in individual who have low levels of vitamin D3 were less physically active. ${ }^{6}$

Clinical features of hyperandrogenism include hirsutism, acne and alopecia in the women with PCOS. In our study, low levels of vitamin D3 associated with higher hirsutism score, that was found to be statistically significant. Wehr et al, also observed negative correlation between serum vitamin D3 levels with hirsutism score who statistically significant had lower levels of vitamin D than non-hirsute women. ${ }^{12}$

In our study, it was observed that acne severity correlated inversely with vitamin D3 levels, like Toossi P et al, studied patients suffering from acne vulgaris including PCOS patients and also found correlation between acne and vitamin D levels but results were not statistically significant. ${ }^{20}$ 
Slyper AH et al studied association of vitamin D with AN and rated AN skin lesions on 4-point scale and their study concluded AN severity was a strong predictor of vitamin D levels. ${ }^{21}$ In this study, also inverse correlation was found between vitamin D3 and severity of AN, all the patients of AN of axilla were vitamin D3 deficient which was found statistically significant. In this study, also AN of neck were divided in 4 grades and vitamin D levels were inversely correlated which was statistically significant.

Obesity is one of the most important features of PCOS, BMI and WHR are well defined parameters to assess it. In this study, also both were found statistically significant inversely correlated with vitamin D3 levels, inferring association between central obesity and vitamin D3 level. Li HW et al, showed inverse association of vitamin D3 level with BMI in PCOS patient with pvalue $<0.05$, in contrast no relationship found in control ovulatory group. ${ }^{15}$ Mahmoudi et al, also reported overweight and obese women with PCOS had significantly decreased levels of vitamin D3 compared with normal weight women with PCOS but that was not found statistically significant. ${ }^{13}$

\section{CONCLUSION}

Recent studies have identified the significant role which vitamin D3 may play in causation of PCOS. In this study, the prevalence of vitamin D3 deficiency in patients of PCOS was found to be $84.3 \%$ which is significant. Various parameters associated with PCOS like waist-hip ratio, obesity, AN of neck and hirsutism score showed positive significant correlation with vitamin D3 deficiency and physical activity of patients of PCOS showed negative correlation with the same. The accumulating data shows correlation between vitamin D3 and PCOS, hence it is speculated that improvement of vitamin D3 levels at a younger age can contribute to prevention of PCOS. There is need for routine vitamin D3 screening in all patients of PCOs for better insight into its role in the syndrome. Screening and correction of vitamin D3 deficiency may prevent PCOS and its manifestations.

Funding: No funding sources

Conflict of interest: None declared

Ethical approval: The study was approved by the Institutional Ethics Committee

\section{REFERENCES}

1. Dunaif A. Insulin Resistance and the Polycystic Ovary Syndrome: Mechanism and Implications for Pathogenesis 1. Endocrine Rev. 1997;18(6):774-800.

2. Diamanti-Kandarakis E, Kouli C, Bergiele A, Filandra F, Tsianateli T, Spina G, et al. A Survey of the Polycystic Ovary Syndrome in the Greek Island of Lesbos: Hormonal and Metabolic Profile. J Clini Endocrinol Metab. 1999;84(11):4006-11.
3. Zawadzki JK, Dunaif A. In:Dunaif A, ed. Polycystic Ovary Syndrome. Boston: Blackwell Scientific, 1995: 377-84.

4. Rotterdam ESHRE/ASRM-Sponsored PCOS Consensus Workshop Group. Revised 2003 consensus on diagnostic criteria and long-term health risks related to polycystic ovary syndrome. Fertil Steril. 2004;81(1):19-25

5. Azziz R, Carmina E, Dewailly D, DiamantiKandarakis E, Escobar-Morreale H, Futterweit W et al. The Androgen Excess and PCOS Society criteria for the polycystic ovary syndrome: the complete task force report. Fertil Steril. 2009;91(2):456-88.

6. Holick M. Vitamin D Deficiency. New England J Medic. 2007;357(3):266-81.

7. Jorde R, Sneve M, Figenschau Y, Svartberg J, Waterloo K. Effects of vitamin D supplementation on symptoms of depression in overweight and obese subjects: randomized double blind trial. J Intrnal Medic. 2008;264(6):599-609.

8. Thys-Jacobs S, Donovan D, Papadopoulos A, Sarrel P, Bilezikian JP. Vitamin D and calcium dysregulation in the polycystic ovarian syndrome. Steroids. 1999;64(6):430-5.

9. Firouzabadi R, Aflatoonian A, Modarresi S, Sekhavat L, Mohammad Taheri S. Therapeutic effects of calcium and vitamin D supplementation in women with PCOS. Complementary Therap Clini Pract. 2012;18(2):85-88.

10. Yildizhan R, Kurdoglu M, Adali E, Kolusari A, Yildizhan B, Sahin $\mathrm{H}$, et al. Serum 25hydroxyvitamin D concentrations in obese and nonobese women with polycystic ovary syndrome. Arch Gynecol Obstet. 2009;280(4):559-63.

11. Hahn S, Haselhorst U, Tan S, Quadbeck B, Schmidt M, Roesler $S$ et al. Low Serum 25-Hydroxyvitamin D Concentrations are Associated with Insulin Resistance and Obesity in Women with Polycystic Ovary Syndrome. Experment Clini Endocrinol Amp Diab. 2006;114(10):577-83

12. Wehr E, Pilz S, Schweighofer N, Giuliani A, Kopera $\mathrm{D}$, Pieber T, et al. Association of hypovitaminosis D with metabolic disturbances in polycystic ovary syndrome. European J Endocrinol. 2009;161(4):57582.

13. Mahmoudi T, Gourabi H, Ashrafi M, Yazdi R, Ezabadi Z. Calciotropic hormones, insulin resistance, and the polycystic ovary syndrome. Fertil Steril. 2010;93(4):1208-14.

14. Lagunova Z, Porojnicu A, ILindberg F, Hexeberg S, Moan J. The Dependency of Vitamin D Status on Body Mass Index, Gender, Age and Season. Anticancer Res. 2009;29(9):3713-20.

15. Li HW, Brereton R, Anderson R, Wallace A, Ho C. Vitamin D deficiency is common and associated with metabolic risk factors in patients with polycystic ovary syndrome. Metab. 2011;60(10):1475-81.

16. Lou Y, Murtola T, Tuohimaa P. Regulation of aromatase and $5 \alpha$-reductase by 25 -hydroxyvitamin D3, 1 $\alpha, 25$-dihydroxyvitamin D3, dexamethasone and 
progesterone in prostate cancer cells. J Steroid Biochem Mol Biol. 2005;94(1-3):151-7.

17. Thomson R, Spedding S, Buckley J. Vitamin D in the aetiology and management of polycystic ovary syndrome. Clin Endocrinol. 2012;77(3):343-50.

18. Figurová J, Dravecká I, Javorský M, Petríková J, Lazúrová I. Prevalence of vitamin D deficiency in Slovak women with polycystic ovary syndrome and its relation to metabolic and reproductive abnormalities. Wiener klinische Wochenschrift. 2015;19:1-8

19. Rashidi B, Haghollahi F, Shariat M, Zayerii F. The Effects of Calcium-Vitamin D and Metformin on Polycystic Ovary Syndrome: A Pilot Study. Taiwanese J Obstet Gynecol. 2009;48(2):142-7.
20. Toossi P, Azizian Z, Yavari H, Fakhim TH, Amini SH, Enamzade R. Serum 25-hydroxy vitamin D levels in patients with acne vulgaris and its association with disease severity. Clini Cases Mineral and Bone Metab. 2015;12(3):238.

21. Slyper AH, Kashmer L, Huang WM, Re'em Y. Acanthosis nigricans, vitamin D, and insulin resistance in obese children and adolescents. J Pediatr Endocrinol Metab. 2014;27(11-12):1107-11.

Cite this article as: Chauhan R, Sahani S, Garg A. Evaluation of vitamin D3 in patients of polycystic ovary syndrome and their correlation. Int J Reprod Contracept Obstet Gynecol 2017;6:2010-6. 